\title{
Continuous and highly ordered organic semiconductor thin films via dip-coating: the critical role of meniscus angle
}

\author{
Xuanyu Liu ${ }^{1}$, Yu Zhang ${ }^{1}$, Xiaotao Zhang ${ }^{1}$, Rongjin $\mathrm{Li}^{1 *}$ and Wenping $\mathrm{Hu}^{1,2,3}$
}

\begin{abstract}
Dip-coating is a low-cost, high-throughput technique for the deposition of organic semiconductors over large area on various substrates. Tremendous studies have been done and many parameters such as withdrawal speed, solvent type and solution concentration have been investigated. However, most of the depositions were ribbons or dendritic crystals with low coverage of the substrate due to the ignorance of the critical role of dynamic solution-substrate interactions during dip-coating. In this study, meniscus angle (MA) was proposed to quantify the real-time in-situ solutionsubstrate interactions during dip-coating. By proper surface treatment of the substrate, the value of MA can be tuned and centimeter-sized, continuous and highly ordered organic semiconductor thin films were achieved. The charge transport properties of the continuous thin films were investigated by the construction of organic field-effect transistors. Maximum (average) hole mobility up to $11.9(5.1) \mathrm{cm}^{2} \mathrm{~V}^{-1} \mathrm{~s}^{-1}$ was obtained. The average mobility was $82 \%$ higher than that of ribbon crystals, indicating the high crystallinity of the thin films. Our work reveals the critical role of dynamic solutionsubstrate interactions during dip-coating. The ability to produce large-area, continuous and highly ordered organic semiconductor thin films by dip-coating could revival the old technique for the application in various optoelectronics.
\end{abstract}

Keywords: organic field-effect transistor, dip-coating, meniscus angle, charge transport, mobility

\section{INTRODUCTION}

Thin films of small-molecule organic semiconductor
(OSC) are intensively studied as the potential channel materials for various optoelectronic devices such as organic field-effect transistors (OFETs), organic memory devices, organic solar cells and organic photodetectors (OPDs) [1-8]. As grain boundaries can significantly reduce the performance of such devices, the ability to deposit continuous and highly ordered OSC thin films is of critical importance for practical applications [1,2,9-14]. Among the various solution-processing methods proposed so far, dip-coating is one of the oldest commercially applied coating techniques. It shows the advantages of low-cost, high-throughput, large-area production, and capability of deposition on curved surfaces, making it a favourable choice for the deposition of thin films of OSCs for optoelectronic applications [15-18].

Over the past decades, great efforts have been made and several key factors that influence the morphology and crystallinity of the deposited thin films of small-molecule OSCs by dip-coating have been investigated (Table S1). For example, the role of withdrawal speed has been systematically investigated by Sele et al [15]. They obtained oriented small grains, needle-shaped crystals and platelets by dip-coating at different withdrawal speeds. $\mathrm{Li}$ et al. $[16,19,20]$ demonstrated the controllable growth of monolayer to multilayer microstripes by dip-coating. They found that the withdrawal speed of the substrate had a significant effect on the thickness and uniformity of the stripes $[16,19,20]$. Chai et al. [21] tried to combine different concentrations and withdrawal speeds to prepare a directed assembly of self-aligned and highly crys-

\footnotetext{
${ }^{1}$ Tianjin Key Laboratory of Molecular Optoelectronic Sciences, Department of Chemistry, School of Science, Tianjin University \& Collaborative Innovation Center of Chemical Science and Engineering, Tianjin 300072, China

${ }^{2}$ Beijing National Laboratory for Molecular Sciences, Key Laboratory of Organic Solids, Institute of Chemistry, Chinese Academy of Sciences, Beijing 100190, China

${ }^{3}$ Joint School of National University of Singapore and Tianjin University, International Campus of Tianjin University, Binhai New City, Fuzhou 350207, China

* Corresponding author (email: lirj@tju.edu.cn)
} 
talline films. Dong et al. [11,22] used mixed solvents to generate Marangoni flow and produced highly oriented ribbon crystals of OSCs. Polymers [23] and their mixture with small-molecule OSCs [24] were also investigated and ribbons were deposited. Recently, Deng et al. [25] reported a strategy that combined microscale photoresist channels with the dip-coating process. The microscale photoresist channels were used to produce a confinement effect to reduce the size of the meniscus. Stripe crystals of both p-type and n-type OSCs were obtained. Although influencing factors such as withdrawal speed $[10,15,16,18,19,21,26-28]$, solvent type $[11,18,29]$ and concentration $[18,21,27,28]$ have been investigated, most of the studies observed the deposition of stripe crystals rather than continuous thin films (Table S1). The key factors that determine the deposition of continuous and highly ordered OSC thin films by dip-coating are still unclear.

Dip-coating is a meniscus-guided solution-deposition technique. In a typical dip-coating process, a target substrate is dipped into the solution and then pulled upward at a controlled speed. During the withdrawal process, a moving meniscus with increased speed of evaporation is formed [23]. The meniscus region is the location where the initiation and continuous deposition of solid films occur [21]. Thus, the shape of the solution meniscus, which relies on the dynamic solution-substrate interactions during dip-coating, has a crucial effect on the morphology and crystallinity of the deposited thin films [1].

Herein, the critical role of dynamic solution-substrate interactions during dip-coating was uncovered. Centimeter-sized, continuous and highly ordered thin films of small-molecule OSCs were obtained by dip-coating for the first time. The strength of dynamic solution-substrate interactions in dip-coating was quantified by the introduction of an angle called meniscus angle (MA). By surface modification of the substrates with self-assembled monolayers (SAMs) of different chemical structures, the MA could be controlled effectively. Transverse mass transport (perpendicular to the withdrawal direction) was enhanced at moderate MA and low withdrawal speed, resulting in the production of continuous and crystalline thin films other than stripe crystals. OFETs based on the continuous thin films showed $82 \%$ increment of mobility compared with those of stripe crystals. Our findings uncover the critical role of dynamic solution-substrate interactions during dip-coating and pave the way for continuous and highly ordered thin films of OSCs produced by dip-coating for various optoelectronic applica- tions.

\section{EXPERIMENTAL SECTION}

\section{Materials}

2,7-Dioctyl[1]benzothieno[3,2-b]benzothiophene (C8BTBT) (Fig. S1a) was purchased from Shanghai Daeyeon (Lumtec). Both phenyltrichlorosilane (PTS, Fig. S1b) and octadecyltrichlorosilane (OTS, Fig. S1c) were purchased from J\&K Scientific Ltd. 6,13-Bis(triisopropylsilylethynyl) pentacene (TIPS-PEN) (Fig. S1d) was purchased from 3A Chemicals. Perylene (Fig. S1e) were purchased from Shanghai Yuanye Bio-Technology Co., Ltd. All chemicals were used without further purification.

\section{Substrate treatment, water contact angle (WCA), and MA measurements}

$\mathrm{SiO}_{2} / \mathrm{Si}$ substrate was cleaned by sonication sequentially in deionized water, acetone and 2-proponol followed by treatment with oxygen plasma at $80 \mathrm{~W}$ for $10 \mathrm{~min}$. The cleaned substrates were immediately modified by PTS or OTS via a vapor phase method. Static WCA and in-situ investigations of MA were recorded and analyzed at room temperature by an OSA-100 Optical Surface Analyzer.

\section{Thin film preparation and characterization}

Highly doped wafers with a $300 \mathrm{~nm}$ thick thermally grown $\mathrm{SiO}_{2}$ dielectric layer were used as the substrate for OFET fabrication. Dip-coating was performed in a clean and closed chamber to reduce the effect of air current and mechanical vibration at room temperature $\left(22-25^{\circ} \mathrm{C}\right)$. The substrate was dipped into an OSC/dichloromethane solution and then withdrawn at a constant rate. The samples were characterized by optical microscope (OptiPlex 3046, Nikon), polarizing microscope (OptiPlex 3046, Nikon) and atomic force microscope (AFM, Dimension Icon). The crystallinity of the thin film was determined by X-ray diffraction (XRD, Rigaku Smartlab) using a $\mathrm{Cu}-\mathrm{Ka}$ radiation source running at $45 \mathrm{kV}$ and $200 \mathrm{~mA}$.

\section{OFET fabrication and characterization}

OFETs were constructed with a bottom-gate, top-contact configuration by stamping $\mathrm{Au}(150 \mathrm{~nm})$ stripes as the source and drain electrodes. Both the ribbons and films were selected with thickness of around $20 \mathrm{~nm}$. Electrical characteristics of the OFETs were measured with a semiconductor parameter analyzer (Keithley 4200-SCS) in ambient air at room temperature. The field-effect mobility $(\mu)$ was calculated in the saturation regime by 
plotting the square root of the drain current versus the gate voltage using $I_{\mathrm{D}}=(W / 2 L) C_{\mathrm{i}} \mu\left(V_{\mathrm{G}}-V_{\mathrm{T}}\right)^{2}$, where $C_{\mathrm{i}}$ is the capacitance per unit area of the gate dielectric layer $\left(C_{\mathrm{i}}=10 \mathrm{nF} \mathrm{cm}{ }^{-2}\right)$, and $W$ and $L$ are the channel width and length, respectively. As for ribbon crystals, the channel width was the sum of the widths of the ribbons between the two electrodes $\left(W=W_{1}+W_{2}+\ldots+W_{n}\right)$ as shown in Fig. S2a.

\section{RESULTS AND DISCUSSION}

C8-BTBT was chosen as the archetype OSC due to its good solubility and excellent mobility [12]. Dichloromethane was selected as the solvent because of its good dissolving capability of small-molecule OSCs, low boiling point, and high evaporation rate at room temperature $[25,26,30,31]$. Three types of substrates, i.e., PTS-modified $\mathrm{SiO}_{2} / \mathrm{Si}$ substrate, OTS-modified $\mathrm{SiO}_{2} / \mathrm{Si}$ substrate and hydroxyl substrate (bared $\mathrm{SiO}_{2} / \mathrm{Si}$ substrate cleaned by oxygen plasma) were utilized to deposit the OSC thin film. The chemical structures of C8-BTBT, PTS and OTS were shown in Fig. S1a-c.

Fig. 1 shows the OM images of the depositions on the three types of substrates obtained at withdrawal speeds $(U)$ of $10,100,700,1000 \mu \mathrm{m} \mathrm{s}^{-1}$, respectively. The concentration of the solution was fixed at $4 \mathrm{mg} \mathrm{mL}^{-1}$. As for OTS substrate, nothing was deposited at all speeds, i.e., the substrates were blank (Fig. 1a-d). At low withdrawal speed of $10 \mu \mathrm{m} \mathrm{s}^{-1}$, discontinuous films appeared on hydroxyl substrate (Fig. 1e), whereas continuous and homogenous films appeared on PTS substrate (Fig. 1i). When the withdrawal speed was increased to $100 \mu \mathrm{m} \mathrm{s}^{-1}$, irregular ribbon arrays appeared on hydroxyl substrate
(Fig. 1f) and regular ribbon arrays appeared on PTS substrate (Fig. 1j). At withdrawal speed of $700 \mu \mathrm{m} \mathrm{s}^{-1}$, leaf textures appeared on both hydroxyl substrate and PTS substrate (Fig. 1g, k). When the withdrawal speed was as high as $1000 \mu \mathrm{m} \mathrm{s}^{-1}$, the molecules could not pack into homogenous films or ribbons on both types of substrates (Fig. 1h, 1). On PTS substrate, continuous thin films were deposited at low withdrawal speeds, while ribbon array crystals were obtained at high withdrawal speeds (Table S2). Under polarized optical microscope (POM), the color of the films (Fig. S3a-c) and ribbon arrays (Fig. S3d-f) changed uniformly when the substrates were rotated by $45^{\circ}$, indicating the absence of grain boundaries and the crystalline nature of the checked films and ribbons. Solutions of different concentrations (2 and $6 \mathrm{mg} \mathrm{mL}^{-1}$ ) were also studied and similar results were found (Figs S4S6).

Fig. 2a and $b$ show the typical AFM images of the ribbon arrays and continuous films on PTS substrate produced at 100 and $10 \mu \mathrm{m} \mathrm{s}^{-1}$, respectively. The thicknesses of the ribbon arrays and thin film were determined to be $23.4 \mathrm{~nm}$ (Fig. 2a) and $22.1 \mathrm{~nm}$ (Fig. 2b), respectively. The root mean square (RMS) roughness of the thin film was $0.48 \mathrm{~nm}$ (Fig. 2b). The atomically flat surface indicated the high crystallinity of the film. Out-of-plane XRD measurements were used to investigate the structure of the depositions produced at different withdrawal speeds from 5 to $700 \mu \mathrm{m} \mathrm{s}^{-1}$ (Fig. 1i-k). As shown in Fig. $2 c$, the peaks were assignable to (00l) diffractions, i.e., (001), (002) and (003) planes with peaks at $2 \theta$ of $3.2^{\circ}, 6.3^{\circ}$ and $9.1^{\circ}$, respectively $[11,32]$. Sharp diffraction peaks appeared for all the depositions (Fig. 1i-k), indicating

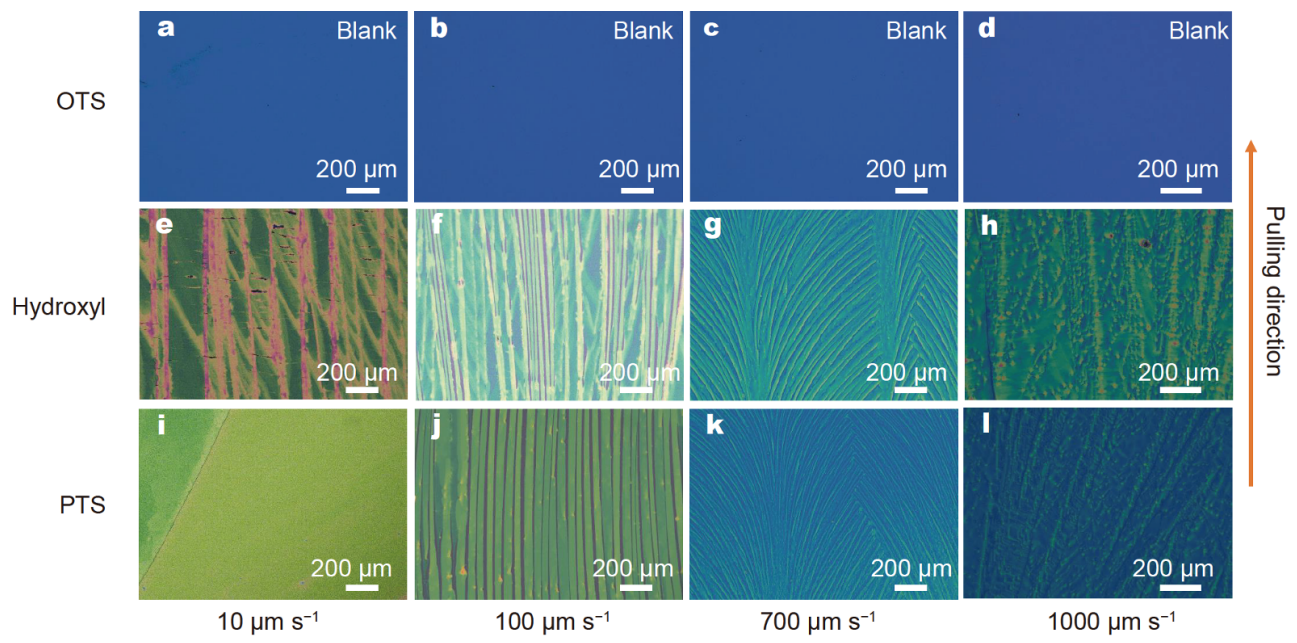

Figure 1 Optical microscopy images of dip-coated C8-BTBT from dichloromethane with OTS substrate (a-d), PTS substrate (i-l) and hydroxyl substrate $(\mathrm{e}-\mathrm{h})$. The withdrawal speeds increase from 10 to $1000 \mathrm{\mu m} \mathrm{s}^{-1}$. 
a
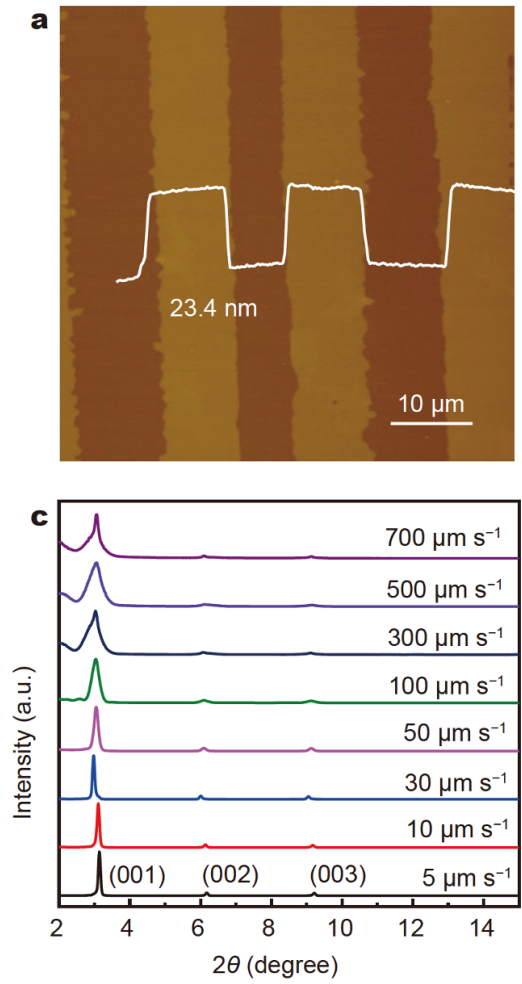

b
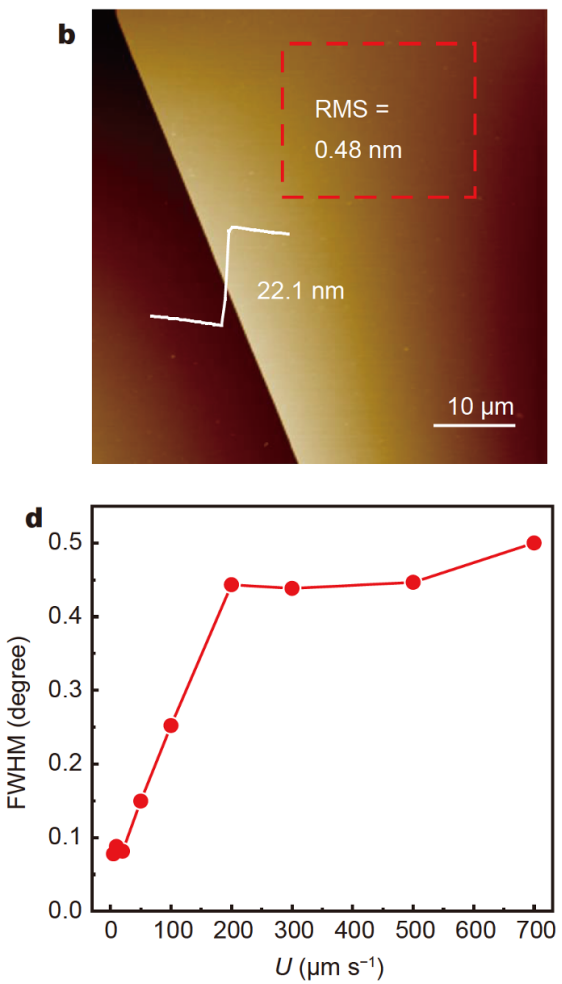

Figure 2 (a, b) AFM images of the ribbon arrays and continuous films. The inset shows the typical thickness of the ribbon and thin film. (c) Out-ofplane XRD of the depositions at different withdrawal speeds $(U)$ from 5 to $700 \mu \mathrm{m} \mathrm{s}^{-1}$. (d) FWHM of the (001) plane as a function of withdrawal speed $(U)$.

their crystalline nature [22]. In-plane XRD results confirmed the crystalline nature of the continuous films on PTS substrate (Fig. S7). Fig. 2d shows the full width at half maximum (FWHM) of the (001) peaks as a function of withdrawal speed $(U)$. When the withdrawal speed was increased from 10 to $200 \mu \mathrm{m} \mathrm{s}^{-1}$, the value of FWHM increased gradually from $0.08^{\circ}$ to $0.43^{\circ}$. At even higher withdrawal speed $\left(>200 \mu \mathrm{m} \mathrm{s}^{-1}\right)$, the value of FWHM was virtually unchanged (Fig. 2d). It is known that the smaller the value of FWHM, the higher the crystal quality $[11,33,34]$. Judging from the XRD measurements, a low withdrawal speed during dip-coating resulted in high crystallinity [35-38].

To elucidate the origin of the different morphologies (Fig. 1), WCA measurements of the three types of substrates (Fig. 3a) were carried out. WCAs of $103.2^{\circ}, 75.9^{\circ}$ and $3.4^{\circ}$ were observed for OTS substrate, PTS substrate and hydroxyl substrate, respectively (Fig. 3b-d), which coincided with previous reports [39] and indicated the successful modification (or treatment) of the substrate. Among these substrates, OTS substrate exhibited the highest dewetting ability and hydroxyl substrate showed the highest wetting ability [40]. Substrates with different

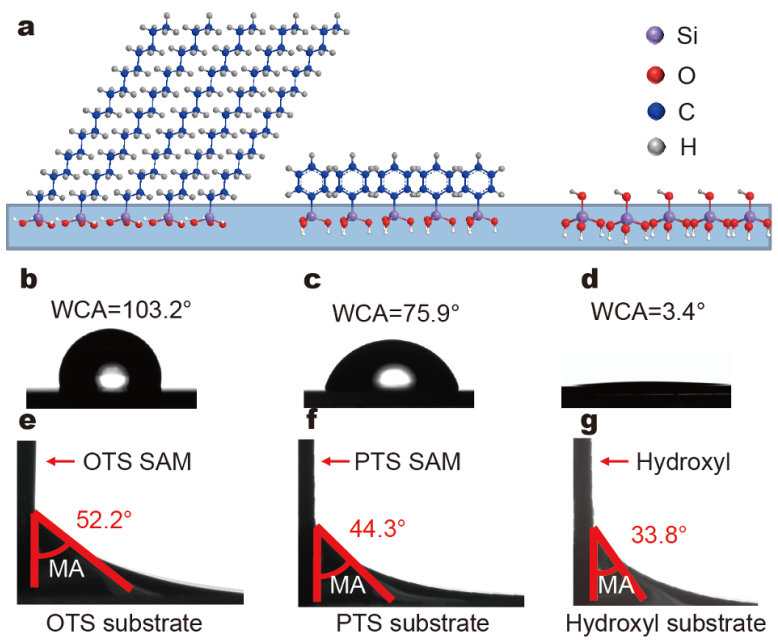

Figure 3 (a) Schematic diagrams of the structures of OTS and PTS selfassembly membranes and the hydroxyl surface. (b-d) WCAs of OTS, PTS and hydroxyl substrates, respectively. (e-g) Photographs of the meniscus regions of OTS, PTS and hydroxyl substrates during dipcoating with the MAs indicated.

WCAs and different surface chemical structures might show different strengths of interactions with the solution during dip-coating. It is known that the curvature of the 
meniscus, which is determined by the surface chemical structures of the substrates and the properties of the solutions, affects the deposition greatly [41]. To quantify the strengths of interactions between the substrates and the solutions during dip-coating, we defined an angle between the withdrawal direction (vertical direction in this study) and the tangential of the curved solution surface of the meniscus when pulling up the substrate as the MA. Clearly, MA indicated the shape of the meniscus during dip-coating. It is known that MA is insensitive to the types of solutes and their concentrations [24], enabling it a quantitative index reflecting the real-time in-situ dynamic solution-substrate interactions during dip-coating. In this study, we found the values of MAs were insensitive to the types of the OSCs and their concentrations (Fig. S8). When OTS substrates were withdrawn from the solution, the MA was as large as $52.2^{\circ}$ (Fig. 3e), which indicated weak interactions between the substrate and the solution [42]. Thus, it was almost impossible for the OTS substrates to entrain the solution and deposit a solid film on the substrate. Indeed, we found no solids on the substrate at withdrawal speed from 10 to $1000 \mu \mathrm{m} \mathrm{s}^{-1}$ (Fig. 1a-d). As for hydroxyl substrate, the MA was as small as $33.8^{\circ}$ (Fig. 3g), indicating a shallow meniscus profile. Strong vertical convection appeared at the direction of gravity during the rapid evaporation of the solvent [43]. As a result, ribbon arrays and leaf textures were obtained at speeds from 10 to $1000 \mathrm{\mu m} \mathrm{s}^{-1}$ (Fig. 1e-h). As for PTS substrate, the MA was about $44.3^{\circ}$, which was between those of the MAs of OTS substrate and hydroxyl substrate (Fig. 3f). With a moderate MA, it was possible for the substrate to entrain the solution during the withdrawal process and there was enough space on the top of the meniscus for the solution self-assembly of the small molecules. Thus, speed-dependent morphologies were possible (Fig. 1i-l).

Depending on the withdrawal speed, there were two different mechanisms of deposition with PTS substrate of moderate MA. When the withdrawal speed was low, the capillarity regime $[41,43]$, which was formed between the substrate and the liquid-air interface, dominated the deposition (Fig. $4 \mathrm{a}, \mathrm{b}$ ). In this case, the movement of the dry line was slow and the solvent evaporation was fast, leading to a continuous supply of the solution to the upper part of the meniscus through capillary rise (Fig. 4a) [44]. Moreover, the profile of the meniscus was thick, providing enough space for both transverse and vertical mass transport (Fig. 4b). The appearance of vertical mass
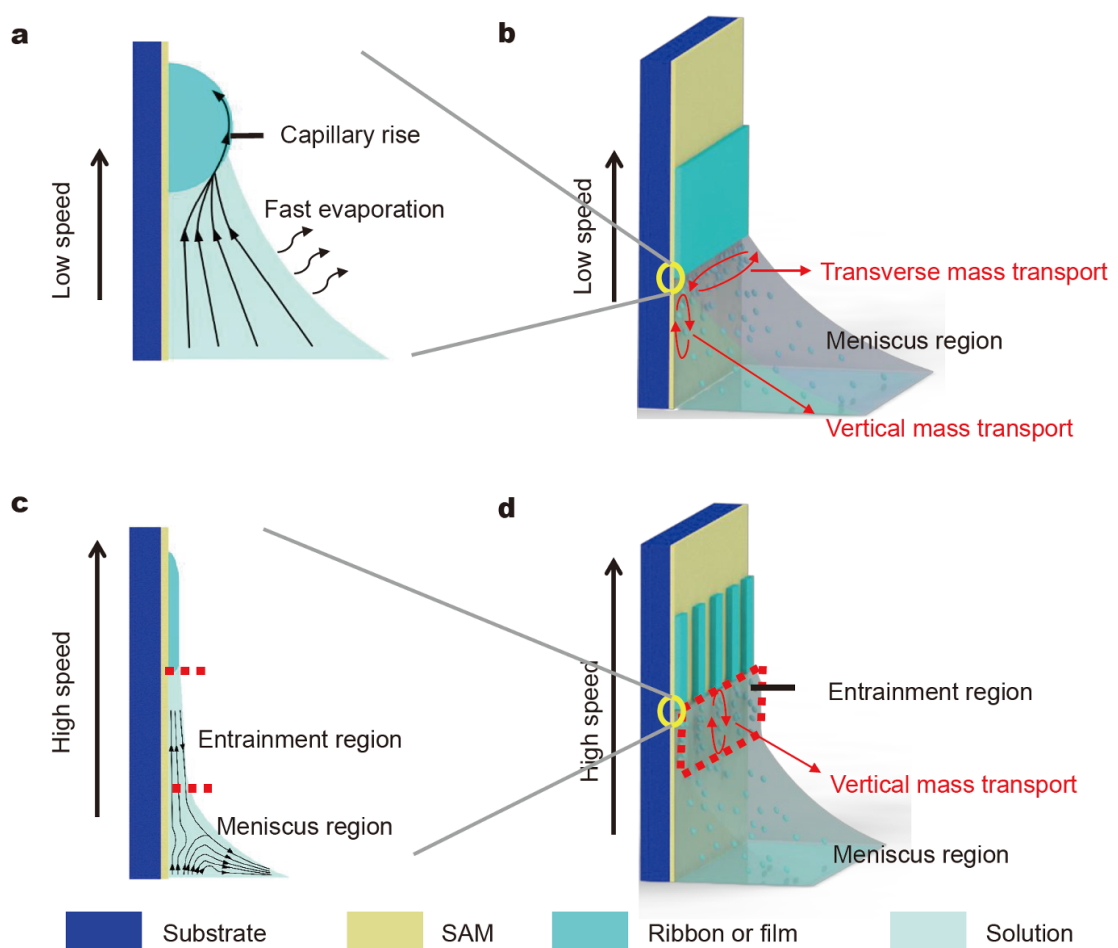

Figure 4 Schematic illustrations of the two different mechanisms of deposition on PTS substrate with moderate MA. (a, c) The liquid-air interface at low and high speed, respectively. (b, d) Mass transport at low and high speed, respectively. At low withdrawal speed, the deposition occurred at the meniscus region with both transverse and vertical mass transport. At high withdrawal speed, the deposition occurred at the entrainment region with only vertical mass transport. 
transport was critical for the deposition of continuous OSC thin films rather than ribbon crystals (Fig. 1i). When the withdrawal speed was high, the deposition was controlled by the entrainment region [41,43], where the solution was entrained on the substrate during the withdrawal process (Fig. 4c, d). The entrainment region exhibited a shallow profile, where transverse mass transport was limited and vertical mass transport was active, resulting in the growth of ribbon crystals (Fig. 1j). To check the general applicability of MA as an index of realtime in-situ solution-substrate interactions for continuous and crystalline films, the depositions of OSCs with different chemical structures were investigated. At moderate MAs on PTS substrate $\left(\sim 44^{\circ}\right.$, Fig. S8), continuous OSC thin films were successfully achieved with perylene and TIPS-PEN at withdrawal speed of $10 \mu \mathrm{m} \mathrm{s}^{-1}$ (Fig. S9a, d). POM images indicated the high crystallinity of the films (Figs S9b, c and S9e, f). On the OTS substrate with a large MA of $52.2^{\circ}$ (Fig. S10a-c), there were almost nothing deposited at withdrawal speed of $10 \mu \mathrm{m} \mathrm{s}^{-1}$ for both perylene and TIPS-PEN. As for the hydroxyl substrate with a small MA of $33.4^{\circ}$ (Fig. S10d-f), the coverage of OSC films on the substrate was low and the films were discontinuous. Solvents such as toluene with high boiling point and small dipole moment were also investigated and confirmed the critical role of MA (Figs S11, S12). Judging from the above results, MA can be used as a quantitative index for the deposition of continuous thin films irrespective of the types of OSCs and solvents.

To investigate the charge transport properties of the continuous and highly ordered thin films, OFETs were constructed. Au stripes (150 nm in thickness) were used as the source and drain electrodes fabricated by a stamping method [45]. PTS-modified $\mathrm{SiO}_{2}(300 \mathrm{~nm})$ was used as the gate dielectric layer. For comparison, OFETs with the same configuration was constructed based on the ribbon arrays. In this structure, the sum of the widths of the ribbons between the channels was used as the channel width (Fig. S2) [46]. Fig. 5a, b show the typical transfer and output characteristics of the devices based on C8BTBT continuous films (the inset shows the OM image of the channel area). The typical transfer and output characteristics of the ribbon arrays are shown in Fig. 5d, e (the inset shows the OM image of the channel area). All devices exhibited typical p-type transfer and output curves in ambient air at room temperature. The mobility distribution of 20 devices of continuous films and ribbon arrays are shown in Fig. 5c, f, respectively. The highest
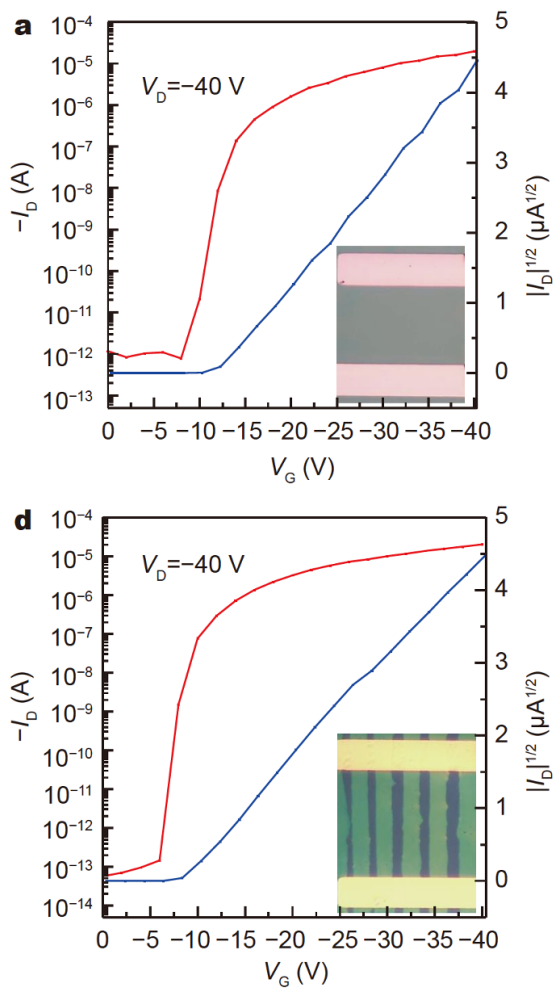
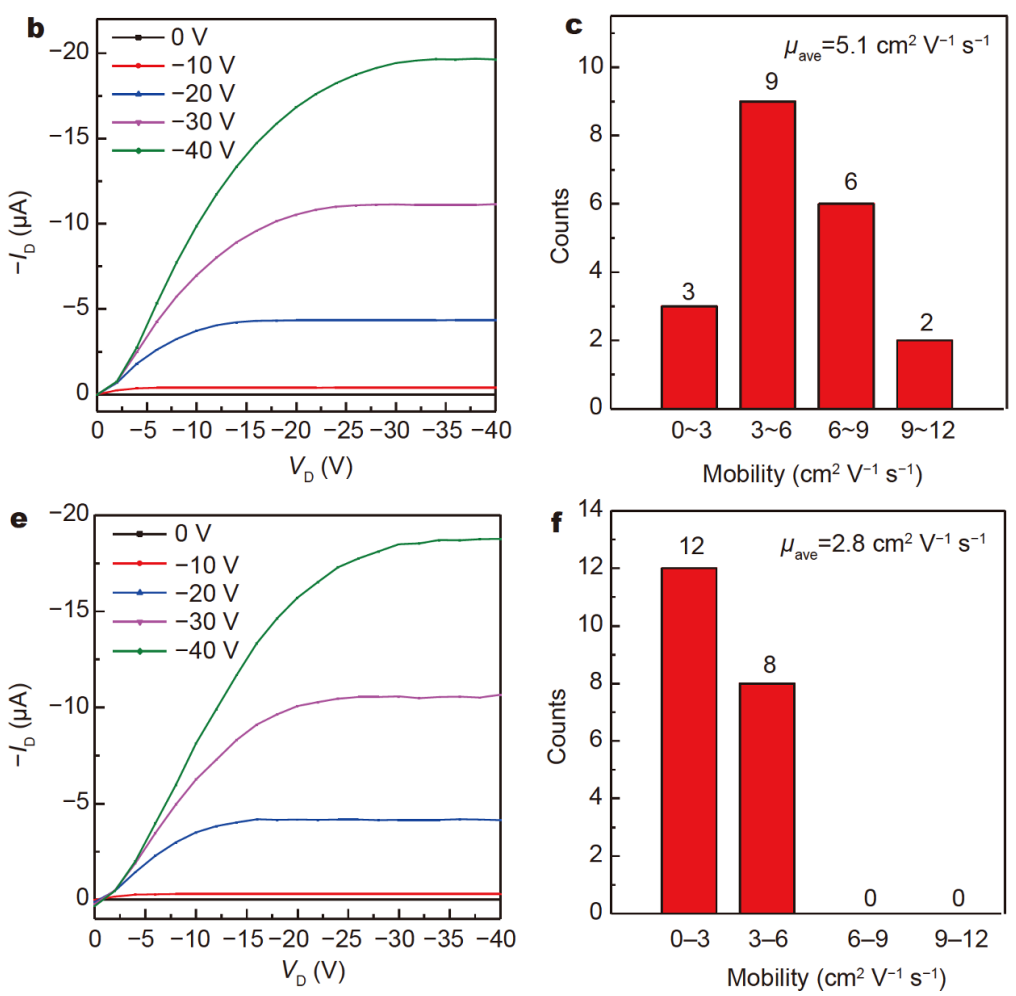

Figure 5 Transfer and output characteristics of C8-BTBT based OFETs fabricated on PTS modified substrate. Transfer and output curves of the continues film (a, b) and ribbon arrays (d, e). (c, f) Histogram of the mobility of 20 C8-BTBT OFETs based on thin films and ribbons. 
(average) mobilities were 11.9(5.1) and $4.6(2.8) \mathrm{cm}^{2} \mathrm{~V}^{-1} \mathrm{~s}^{-1}$ for continuous films and ribbon arrays, respectively. The average mobilities of the continuous films were $82 \%$ higher than those of ribbon arrays. Judging from the XRD measurements, the films showed a smaller value of FWHM (Fig. 2c), indicating the higher crystallinity (i.e., higher order of molecular packing in the solids) compared with that of the ribbons. It was known that the higher the crystallinity, the higher the mobility. The high mobility based on the continuous films further proved the usefulness of dip-coating as an efficient technology to deposit thin films of OSCs for various optoelectronic applications.

\section{CONCLUSIONS}

In summary, MA could be used as an index to quantify the real-time in-situ dynamic solution-substrate interactions during dip-coating. A moderate $\mathrm{MA}$ and low withdrawal speed enhanced vertical mass transport in the meniscus region and resulted in the production of large area, continuous and highly ordered organic semiconductor thin films.

\section{Received 1 February 2020; accepted 10 March 2020; published online 23 April 2020}

1 Diao Y, Shaw L, Bao Z, et al. Morphology control strategies for solution-processed organic semiconductor thin films. Energy Environ Sci, 2014, 7: 2145-2159

$2 \mathrm{Gu} \mathrm{X}$, Shaw L, Gu K, et al. The meniscus-guided deposition of semiconducting polymers. Nat Commun, 2018, 9: 534

3 Qian J, Jiang S, Li S, et al. Solution-processed 2D molecular crystals: Fabrication techniques, transistor applications, and physics. Adv Mater Technol, 2018, 4: 1800182

4 Yang F, Cheng S, Zhang X, et al. 2D organic materials for optoelectronic applications. Adv Mater, 2017, 30: 1702415

5 Yao Y, Zhang L, Leydecker T, et al. Direct photolithography on molecular crystals for high performance organic optoelectronic devices. J Am Chem Soc, 2018, 140: 6984-6990

6 Jiang $\mathrm{H}$, Zhao $\mathrm{H}$, Zhang KK, et al. High-performance organic single-crystal field-effect transistors of indolo[3,2-b]carbazole and their potential applications in gas controlled organic memory devices. Adv Mater, 2011, 23: 5075-5080

7 Usta H, Kim D, Ozdemir R, et al. High electron mobility in [1] benzothieno[3,2-b][1]benzothiophene-based field-effect transistors: Toward n-type BTBTs. Chem Mater, 2019, 31: 5254-5263

8 Schünemann C, Wynands D, Eichhorn KJ, et al. Evaluation and control of the orientation of small molecules for strongly absorbing organic thin films. J Phys Chem C, 2013, 117: 11600-11609

9 Jeong H, Han S, Baek S, et al. Dense assembly of soluble acene crystal ribbons and its application to organic transistors. ACS Appl Mater Interfaces, 2016, 8: 24753-24760

10 Wang W, Wang L, Dai G, et al. Controlled growth of large-area aligned single-crystalline organic nanoribbon arrays for transistors and light-emitting diodes driving. Nano-Micro Letters, 2017, 9: 52

11 Wang Z, Guo H, Li J, et al. Marangoni effect-controlled growth of oriented film for high performance C8-BTBT transistors. Adv Mater Interfaces, 2019, 6: 1801736

12 Yuan Y, Giri G, Ayzner AL, et al. Ultra-high mobility transparent organic thin film transistors grown by an off-centre spin-coating method. Nat Commun, 2014, 5: 3005

13 Jiang H, Zhang KK, Ye J, et al. Atomically flat, large-sized, twodimensional organic nanocrystals. Small, 2013, 9: 990-995

14 Nikolka M, Nasrallah I, Rose B, et al. High operational and environmental stability of high-mobility conjugated polymer fieldeffect transistors through the use of molecular additives. Nat Mater, 2017, 16: 356-362

15 Sele CW, Kjellander BKC, Niesen B, et al. Controlled deposition of highly ordered soluble acene thin films: Effect of morphology and crystal orientation on transistor performance. Adv Mater, 2009, 21: 4926-4931

16 Li L, Gao P, Wang W, et al. Growth of ultrathin organic semiconductor microstripes with thickness control in the monolayer precision. Angew Chem Int Ed, 2013, 52: 12530-12535

17 Liu N, Ma W, Tao J, et al. Cable-type supercapacitors of threedimensional cotton thread based multi-grade nanostructures for wearable energy storage. Adv Mater, 2013, 25: 4925-4931

$18 \mathrm{Wu} \mathrm{K}, \mathrm{Li} \mathrm{H}, \mathrm{Li} \mathrm{L}$, et al. Controlled growth of ultrathin film of organic semiconductors by balancing the competitive processes in dip-coating for organic transistors. Langmuir, 2016, 32: 6246-6254

19 Li L, Gao P, Schuermann KC, et al. Controllable growth and fieldeffect property of monolayer to multilayer microstripes of an organic semiconductor. J Am Chem Soc, 2010, 132: 8807-8809

20 Li L, Gao P, Baumgarten M, et al. High performance field-effect ammonia sensors based on a structured ultrathin organic semiconductor film. Adv Mater, 2013, 25: 3419-3425

21 Chai Z, Abbasi SA, Busnaina AA. Scalable directed assembly of highly crystalline 2,7-dioctyl[1]benzothieno[3,2-b][1]benzothiophene (C8-BTBT) films. ACS Appl Mater Interfaces, 2018, 10: 18123-18130

22 Zhao H, Wang Z, Dong G, et al. Fabrication of highly oriented large-scale tips pentacene crystals and transistors by the marangoni effect-controlled growth method. Phys Chem Chem Phys, 2015, 17: 6274-6279

23 Ghosh M, Fan F, Stebe KJ. Spontaneous pattern formation by dip coating of colloidal suspensions on homogeneous surfaces. Langmuir, 2007, 23: 2180-2183

24 Zhang K, Marszalek T, Wucher P, et al. Crystallization control of organic semiconductors during meniscus-guided coating by blending with polymer binder. Adv Funct Mater, 2018, 28: 1805594

25 Deng W, Zhang X, Dong $\mathrm{H}$, et al. Channel-restricted meniscus self-assembly for uniformly aligned growth of single-crystal arrays of organic semiconductors. Mater Today, 2019, 24: 17-25

26 Nam S, Jeong YJ, Jung J, et al. Direct printing of soluble acene crystal stripes by a programmed dip-coating process for organic field-effect transistor applications. J Mater Chem C, 2018, 6: 799807

27 Zheng $\mathrm{S}$, Xiong $\mathrm{X}$, Zheng $\mathrm{Z}$, et al. Solution-grown large-area $\mathrm{C}_{60}$ single-crystal arrays as organic photodetectors. Carbon, 2018, 126: 299-304

28 Yang Z, Song P, Feng F, et al. Influence of dip-coating temperature upon film thickness in chemical solution deposition. IEEE Trans Appl Supercond, 2018, 28: 7500205

29 Takahashi $\mathrm{K}$, Shan $\mathrm{B}, \mathrm{Xu} \mathrm{X}$, et al. Engineering thin films of a tetrabenzoporphyrin toward efficient charge-carrier transport: Selective formation of a brickwork motif. ACS Appl Mater Interfaces, 
2017, 9: 8211-8218

30 Xu X, Deng W, Zhang X, et al. Dual-band, high-performance phototransistors from hybrid perovskite and organic crystal array for secure communication applications. ACS Nano, 2019, 13: 5910-5919

31 Jang J, Nam S, Im K, et al. Highly crystalline soluble acene crystal arrays for organic transistors: Mechanism of crystal growth during dip-coating. Adv Funct Mater, 2012, 22: 1005-1014

32 Ebata H, Izawa T, Miyazaki E, et al. Highly soluble [1]benzothieno $[3,2-b]$ benzothiophene (BTBT) derivatives for high-performance, solution-processed organic field-effect transistors. J Am Chem Soc, 2007, 129: 15732-15733

33 Yu X, Yu J, Zhou J, et al. Organic field-effect transistors with a sandwich structure from inserting 2,2',2"-(1,3,5-benzenetriyl)tris [1-phenyl-1 $h$-benzimidazole] in the pentacene active layer. Eur Phys J Appl Phys, 2013, 62: 20101

34 Wei W, Yang C, Mai J, et al. High mobility solution-processed $\mathrm{C}_{8}$ BTBT organic thin-film transistors via UV-ozone interface modification. J Mater Chem C, 2017, 5: 10652-10659

35 Izawa T, Miyazaki E, Takimiya K. Molecular ordering of highperformance soluble molecular semiconductors and re-evaluation of their field-effect transistor characteristics. Adv Mater, 2008, 20: 3388-3392

36 Liu C, Minari T, Lu X, et al. Solution-processable organic single crystals with bandlike transport in field-effect transistors. Adv Mater, 2011, 23: 523-526

37 Gbabode G, Dohr M, Niebel C, et al. X-ray structural investigation of nonsymmetrically and symmetrically alkylated [1]benzothieno $[3,2-b]$ benzothiophene derivatives in bulk and thin films. ACS Appl Mater Interfaces, 2014, 6: 13413-13421

38 Zhang Z, Peng B, Ji X, et al. Marangoni-effect-assisted bar-coating method for high-quality organic crystals with compressive and tensile strains. Adv Funct Mater, 2017, 27: 1703443

39 Janssen D, De Palma R, Verlaak S, et al. Static solvent contact angle measurements, surface free energy and wettability determination of various self-assembled monolayers on silicon dioxide. Thin Solid Films, 2006, 515: 1433-1438

40 Guo DY, Tsai Y, Yu TF, et al. Interfacial effects on solutionsheared thin-film transistors. J Mater Chem C, 2018, 6: 1200612015

41 Kim O, Nam J. Confinement effects in dip coating. J Fluid Mech, 2017, 827: 1-30

42 Casalini S, Bortolotti CA, Leonardi F, et al. Self-assembled monolayers in organic electronics. Chem Soc Rev, 2017, 46: 40-71

43 Corrales TP, Bai M, del Campo V, et al. Spontaneous formation of nanopatterns in velocity-dependent dip-coated organic films: from dragonflies to stripes. ACS Nano, 2014, 8: 9954-9963

44 Brinker CJ. Dip coating. In: Schneller T, Schneller R, Kosec M, Payne D (eds.). Chemical Solution Deposition of Functional Oxide Thin Films. Berlin: Springer, 2013, 233-261

45 Tang Q, Tong Y, Li H, et al. High-performance air-stable bipolar field-effect transistors of organic single-crystalline ribbons with an air-gap dielectric. Adv Mater, 2008, 20: 1511-1515

46 Zhu X, Wang Q, Tian X, et al. Unidirectional and crystalline organic semiconductor microwire arrays by solvent vapor annealing with PMMA as the assisting layer. J Mater Chem C, 2018, 6: 12479-12483

Acknowledgements The authors acknowledge financial support from the National Natural Science Foundation of China (51873148, 61674116 and 51633006), the Ministry of Science and Technology of China (2016YFA0202302) and the Natural Science Foundation of Tianjin (18JC-YBJC18400)

Author contributions $\mathrm{Li} \mathrm{R}$ and $\mathrm{Hu} \mathrm{W}$ conceived the idea and directed the project. Liu X carried out most of the experiments. Zhang Y performed some OFETs measurements. Zhang $\mathrm{X}$ performed the XRD measurements. Liu X and Li R wrote the paper. All authors analyzed the experimental results and contributed to the discussion.

Conflict of interest The authors declare no conflict of interest.

Supplementary information Experimental details and supporting data are available in the online version of the paper

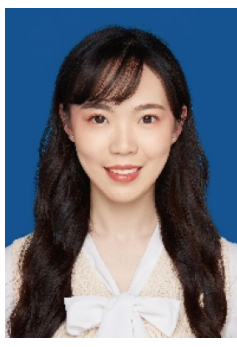

Xuanyu Liu obtained her bachelor degree from Zhengzhou University in 2017. She is currently a graduate student in the School of Science, Tianjin University. Her main research interests are the solution processing of OSCs and their optoelectronic properties.

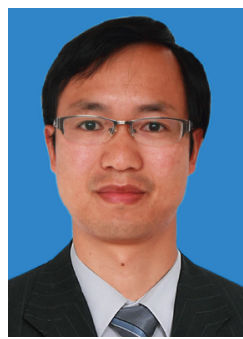

Rongjin Li is a Professor of Tianjin University. $\mathrm{He}$ received his $\mathrm{PhD}$ from the Institute of Chemistry, Chinese Academy of Sciences (CAS) in 2009 supervised by Prof. Wenping Hu. His research interests include OSCs, two dimensional molecular crystals and organic optoelectronic devices.

\section{浸渍提拉法制备连续、高度有序有机半导体薄膜: 弯月面角的关键作用}

刘璇宇 ${ }^{1}$, 张钰 ${ }^{1}$, 张小涛 ${ }^{1}$, 李荣金 ${ }^{*}$, 胡文平 ${ }^{1,2,3}$

摘要 浸渍提拉法是一种低成本、大面积、高效制备有机半导体 薄膜的技术. 针对该技术中提拉速度、溶剂类型、溶液浓度等参 数的研究报道己很多. 但由于忽略了提拉过程中溶液-衬底相互作 用这个关键因素, 提拉得到的沉积物绝大多数为带状或树枝状晶 体, 覆盖率较低. 本文提出用弯月面角(MA)来量化浸渍提拉过程 中溶液与衬底的实时相互作用的强弱. 通过对衬底进行适当的表 面处理, 可以调节MA值, 从而可以获得厘米级、连续、高度有序 的有机半导体薄膜, 并构建了有机场效应晶体管(OFETs), 研究了 连续薄膜的电荷输运特性. 最大 (平均) 空穴迁移率可达 $11.9(5.1) \mathrm{cm}^{2} \mathrm{~V}^{-1} \mathrm{~s}^{-1}$. 统计显示连续薄膜的平均迁移率比带状晶体 高 $82 \%$ ，表明所制备的薄膜具有高结晶度. 该工作揭示了浸渍提拉 过程中动态溶液-衬底相互作用在薄膜沉积过程中扮演的关键角 色, 给出了衡量二者之间相互作用强弱的量化指标, 为浸渍提拉法 制备大面积、连续、高度有序的有机半导体薄膜的研究及其在各 种光电器件领域的应用提供了理论和技术支撑. 